\title{
Interação natural como apoio a atividade de telementoria em Telemedicina
}

\author{
Anna Carolina Soares Medeiros ${ }^{1}$, Rafael Henrique Assis de Castro ${ }^{1}$, Danielle \\ Rousy Dias da Silva ${ }^{1,2}$, Tatiana Aires Tavares ${ }^{1,2}$ \\ ${ }^{1}$ Centro de Informática - Universidade Federal da Paraíba (UFPB) \\ Campus Universitário Castelo Branco - João Pessoa- PB - Brasil \\ ${ }^{2}$ Laboratório de Aplicações de Vídeo Digital (LAVID) - UFPB \\ Campus Universitário Castelo Branco - João Pessoa- PB - Brasil \\ linnamedeiros@gmail.com, rafaelhenriquecegmail.com, \\ tatiana@lavid.ufpb.br, danielle@ci.ufpb.br
}

\begin{abstract}
Telementoring is bringing new trends in classrooms, especially in regard to training and distance learning. Tutors have a goal of exchanging experiences and sharing their professional experiences with students. In telemedicine, characterized by the use of Information and Communication Technologies for Health, telementoring is even more interesting because it expands the ability of specialized training and qualifying medical residencies. This paper presents the initial results from the use of Natural Interaction to support activity in a virtual video collaboration environment supported by Arthron tool. We also detail the development of a Natural Interaction module as an alternative for the control of $3 D$ objects in this developed environment.
\end{abstract}

Resumo. A telementoria está trazendo novos recursos em salas de aula, especialmente no que tange a capacitação e ensino a distância. Os tutores têm por meta a troca de experiências e o compartilhamento da sua vivência profissional com os alunos. Na Telemedicina, caracterizada pela utilização de Tecnologias de Informação e Comunicação para Saúde, a telementoria é ainda mais interessante, pois expande a capacidade de treinamentos especializados e qualifica residências médicas. Neste artigo são apresentados os resultados iniciais da utilização de Interação Natural como apoio a atividade de telementoria em um ambiente de vídeo colaboração em Saúde suportado pela ferramenta Arthron. Mais especificamente, é detalhado o desenvolvimento de um módulo de Interação Natural como forma alternativa para o controle de objetos $3 D$ neste ambiente.

\section{Introdução}

Na comunicação humana sempre se utilizou de gestos, expressões e movimentos como suporte à linguagem oral. Certos gestos são tão comumente utilizados pelo mundo, que são compreendidos por diferentes culturas e, inclusive, em épocas distintas, como pode ser visto nos gestos analisados em (McCord, 1948).

$\mathrm{Na}$ comunicação humana mediada por computadores não é tão diferente. Desde os primeiros exemplares tentou-se pensar na criação de dispositivos que se aproximassem de gestos feitos no dia-a-dia, facilitando assim o aprendizado dos mesmos por meio de algum grau de intuitividade. Fazendo uma retrospectiva, podemos citar o mouse como 
exemplo de avanço na Interação Humano Computador (Carroll, 2009); mais adiante, o surgimento das telas sensíveis ao toque, da utilização de câmeras na análise das ações do usuário e da criação de dispositivos que nos permitem o uso da tecnologia com mais facilidade. A exemplo disso tem-se o Kinect, podemos vislumbrar a contribuição desse e outros artefatos tecnológicos no desenvolvimento de interfaces de usuário, que possibilitem uma aproximação cada vez maior com a interação natural.

Interação Natural (IN) é considerada um conceito que estuda formas do homem interagir com dispositivos eletrônicos através dos cinco sentidos do ser humano, seja através de gestos, comandos de voz, movimentos e expressões corporais ou detecção e identificação de partes do corpo humano como rosto, mão, polegar, retina, articulações, entre outros (Valli, 2007). A Interação Natural ganhou popularidade com o advento dos jogos movidos a gestos a exemplo do Nintendo Wii, cujos controles permitem uma interação diferente dos "antigos" consoles, não só apertando botões, mas apontando, mexendo, girando, oferecendo um mundo de novas interações. A partir da inovação da Nintendo, surgiram variações como o PlayStation Move da Sony que funciona com o Playstation3, Já Microsoft trouxe com o Kinect ainda mais inovação, uma vez que permite o uso do próprio corpo e seus movimentos para interação (Yonk, 2010).

A atividade de telementoria (do inglês tele-mentoring) está trazendo novos recursos em salas de aula e resgatando a figura do aprendiz na relação professor-aluno. Os professores que utilizam estratégias de mentoria não se restringem a relação unidirecional de fornecer informações e apontadores, eles têm por meta a troca de experiências e o compartilhamento da sua vivência profissional. Na área da Saúde, esse tipo de atividade é essencial ao aprendizado, como destaca a professora e psiquiatra Emirene Maria Trevisan Navarro da Cruz, coordenadora do Programa Tutoria (Mentoring) da FAMERP; também na escola médica, um tutor, na acepção ampla de "cuidador", pode ser útil provendo ao aluno-médico uma figura modelo-suporte para auxiliá-lo nas vicissitudes do processo de vir-a-ser médico e cidadão (Sartori, 2004).

Dessa forma, a utilização de recursos e interfaces cada vez mais sofisticadas para apoio a telementoria torna possível que o compartilhamento de experiências reais, como acompanhamento de uma cirurgia, preserve toda sua riqueza de informação no mundo virtual através de ambientes mediados pelo computador. A ferramenta Arthron (Silva, 2011) oferece um ambiente de vídeo-colaboração em Saúde para Telemedicina, voltada inicialmente para transmissão de cirurgias em tempo-real (Oliveira, 2012), a Arthron está buscando a integração de novas tecnologias para enriquecer a atividade de telementoria, tornando a experiência do aprendiz mais realista e interativa.

Nesse sentido, o presente trabalho apresenta os primeiros resultados visando a integração de técnicas de Interação Natural como apoio à atividade de telementoria. Para tanto, um módulo de IN, utilizando o Kinect, foi acoplado à Arthron, como recurso adicional para manipulação de modelos 3D. Uma das novas facetas da Arthron é a possibilidade de manipular objetos 3D, especialmente estruturas anatômicas humanas, durante a transmissão dos fluxos de áudio e vídeo (oriundos da transmissão da cirurgia). Neste trabalho, discutimos como aplicar técnicas de IN para facilitar a manipulação desses objetos e, inclusive, viabilizar esse tipo de interação, dentro do centro cirúrgico, onde o médico-tutor, poderá manipular os objetos livre de contaminação manual. 


\section{Interação Natural na área da Saúde}

É certo que as capacidades físicas e mentais do usuário de uma determinada interface são consideradas em qualquer projeto, pois, o perfil do usuário influencia o desenvolvimento da interface. De uma forma geral, as pessoas naturalmente se comunicam através de gestos, expressões e movimentos; desenvolver aplicações que lidam com Interação Natural significa criar sistemas capazes de entender tais ações dos usuários enquanto eles estão interagindo naturalmente com o ambiente em sua volta. Portanto, as pessoas não precisam vestir ou usar algum equipamento, muito menos aprender instruções específicas, pois a interação é intuitiva. Interface natural segue um novo paradigma com o objetivo de respeitar a percepção humana (Valli, 2007).

A dimensão lúdica está sempre presente na interação natural o que é especialmente interessante quando queremos representar e reproduzir a realidade a distância. No caso especial da telementoria, essa característica representa o poder de captar a atenção de dos aprendizes de modo que eles não se preocupem com a fadiga e não fiquem facilmente entediados. Essa é a grande vantagem da implementação da interação natural em diversas aplicações.

O Kinect é uma tecnologia de interação natural desenvolvida pela Microsoft que utiliza uma câmera 3D e software de reconhecimento de gestos para deixar as pessoas jogarem videogames utilizando movimentos naturais do corpo, em vez de segurar algum controle específico (Chapman, 2010). Essa tecnologia tem o propósito inicial de dar suporte ao console da Microsoft Xbox 360 para possibilitar essa nova forma de se jogar em vários tipos de games, porém, recentemente, pesquisas apontam novos rumos para Kinect que já está sendo utilizado com outros fins, como por exemplo, navegar no Google Earth utilizando movimentos naturais (Yin \& Davis, 2010). Além desse cenário, podemos citar a utilização de tecnologias de IN em salas de cirurgia, utilizando gestos para controlar os programas, no acesso a imagens da cirurgia; tudo isso sem exigir que o médico tenha contato com nenhum hardware adicional, protegendo dessa forma o paciente contra possíveis infecções (Strickland, Tremaine, \& Brigley, 2011).

Outra área da Saúde que tem se beneficiado da IN é a Fisioterapia, tornando exercícios enfadonhos e repetitivos mais cativantes para o paciente. (Kizony, 2003) apresenta um sistema em realidade virtual voltado para reabilitação de pacientes com algum tipo de deficiência motora; (Tanner, 2008) apresenta um jogo desenvolvido utilizando o Wiimote que visa tornar os exercícios da fisioterapia mais atrativos e envolventes; (Miller, 2007) apresenta um estudo que mostra resultados na aceleração do processo de reabilitação com o uso do videogame Wii; (Vidal, 2011) apresenta uma solução para reabilitação de pacientes com o uso do Kinect para atuação em exercícios que visam a avaliação do equilíbrio em pacientes idosos.

Podemos perceber que o desenvolvimento tecnológico promove o acesso constante à informação e impõe modificações profundas em todas as áreas do conhecimento, apresentando necessidades urgentes de pesquisas que evidenciem a evolução da interface homem-computador no sentido de acompanhar esse desenvolvimento. Numa área crítica como a Saúde, o desempenho humano no uso de computadores e de sistemas de informação vem se expandindo consideravelmente. Sistemas de Telemedicina voltados para virtualização da sala de cirurgia, não apenas exigem desenvolvimento tecnológico, como também exigem a adequação do profissional de Saúde para interagir com determinados artefatos tecnológicos, à distância. É um cenário 
que exige aspectos de usabilidade relacionados à cognição humana. Por isso, o uso de interfaces inovadoras tem papel primordial nessa etapa de apropriação de novas tecnologias, uma vez que por ela, médicos, aprendizes e professores passarão a se comunicar e a interagir com a aplicação.

\section{Desenvolvimento da interface em IN na Arthron}

A experiência apresentada neste trabalho envolve o desenvolvimento e a avaliação de uma interface de usuário baseada em IN para manipulação 3D na ferramenta Arthron (Silva, 2011). A Arthron é uma ferramenta voltada para prover facilidades para vídeo colaboração em Saúde que possibilita além de fluxos de mídia, como vídeo, utilizar recursos tridimensionais. A Arthron tem por principal funcionalidade oferecer ao usuário uma interface simples para manipulação de diferentes fontes/fluxos de mídia simultâneos. Dessa forma o usuário pode remotamente adicionar, remover, configurar o formato de apresentação e programar a exibição no tempo e no espaço dos fluxos de mídia. A ferramenta é composta pelos seguintes componentes: Articulador (Manager), Agente Codificador (Encoder), Agente Decodificador (Decoder), Refletor (Distribuidor de fluxo), Servidor de Vídeo (VideoServer), Servidor de Gerenciamento do Usuário (WebService) e o Agente para Videoconferência (VideoRoom).

Os fluxos gerenciados pela Arthron podem ser gerados em localizações geograficamente distribuídas. A ferramenta é responsável por capturar, controlar, transcodificar, transmitir e decodificar as mídias capturadas em tempo real ou de arquivo. Essas mídias podem ser enviadas em alta, média e baixa definição, simultaneamente, tanto para decodificadores específicos na rede quanto para a Internet. Na Fig. 1 podemos observar a tela da Arthron que permite o controle de múltiplos fluxos de vídeo simultâneos durante a transmissão de uma cirurgia. É possível observar os codificadores (janela superior) e os decodificadores (janela inferior).

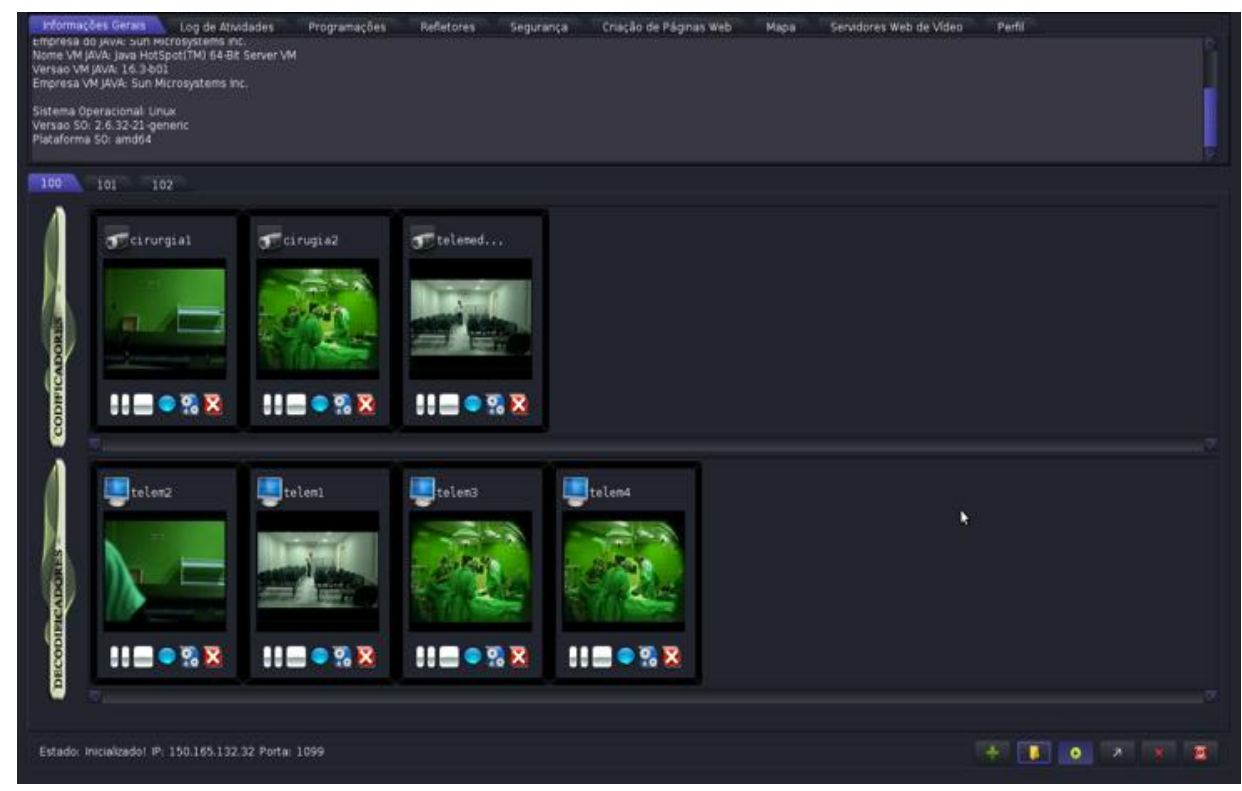

Figura 1. Interface de Usuário da Arthron para Controle de Fluxos de Vídeo.

Um exemplo de uso da Arthron hoje pode ser observado na Fig. 2. Nesta figura observamos uma aula ministrada na sala de telemedicina do HULW (Hospital Universitário Lauro Wanderley). Em (a) podemos observar a projeção do slide utilizado 
como recurso didático padrão para aula de Cirurgia. Em (b) são observados as imagens capturadas ao vivo pela Arthron direto do centro cirúrgico do HULW. O tutor (médico cirurgião) está no centro cirúrgico executando a cirurgia e, enquanto isso, pode interagir com o professor (médico responsável) e os demais aprendizes na sala de Telemedicina através do uso da Arthron. Nesse contexto, a telementoria visa preparar estudantes para desenvolver tarefas reais, oferecer a profissionais meios de treinar novas técnicas com a colaboração de profissionais a distância.

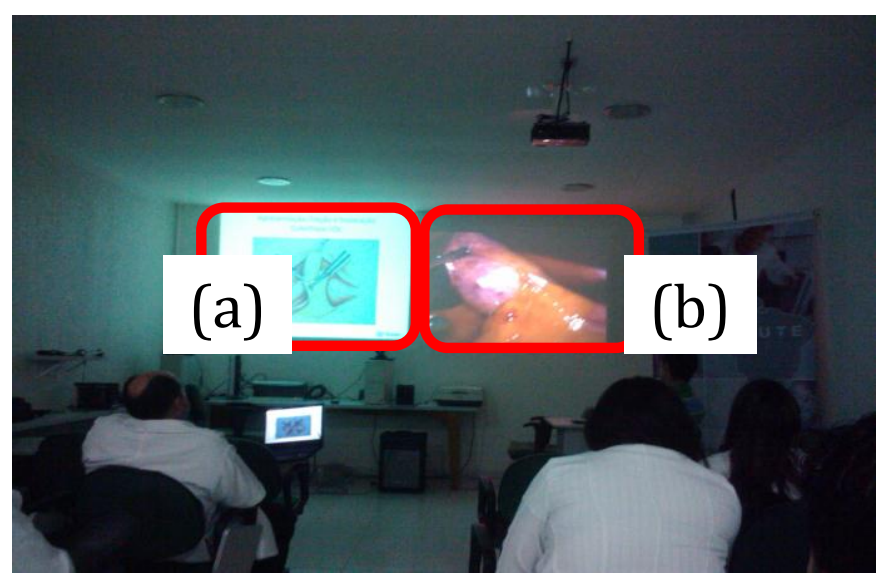

Figura 2. Arthron utilizada para telementoria no HULW-UFPB..

A educação e o treinamento são os temas mais explorados no contexto das aplicações da Realidade Virtual (RV), pois permitem uma interação humano-computador mais natural em ambiente 3D e possibilitar a reprodução de situações reais, tornando-se um recurso de amplo potencial para a área da Saúde (Nunes et al., 2011).

Logo, a adição desse tipo de recurso à Arthron foi uma tendência natural. A adição de modelos 3D de estruturas anatômicas humanas é especialmente útil como recurso didático voltado à atividade de telementoria proporcionando uma experiência mais completa de ensino. Através dessa funcionalidade o médico-professor pode mostrar aos aprendizes de forma integrada ao vídeo ao vivo modelos que demonstram o funcionamento normal de órgãos, tecidos ou estruturas do corpo humano como pode ser visto na Fig. 3.

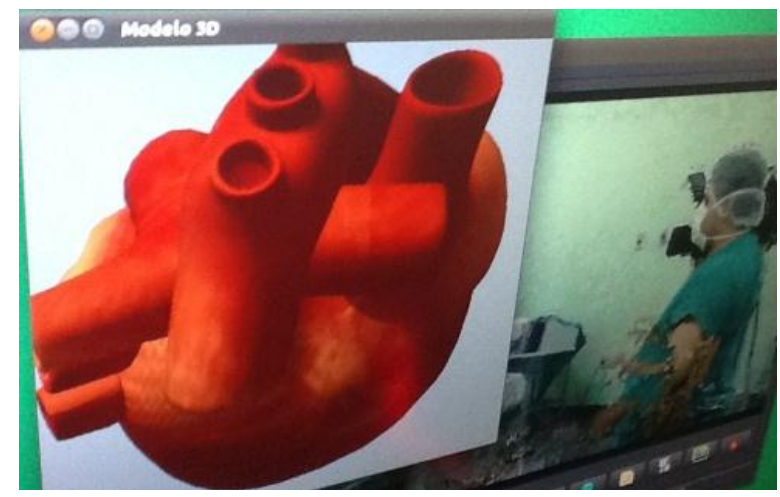

Figura 3. Integração de Objetos 3D na Arthron.

No entanto, as interfaces tridimensionais de modo geral exigem a operação de comandos complexos ou dispositivos de interação especiais (joysticks) para sua manipulação. Dessa forma, o que seria um benefício poderia se tornar uma sobrecarga para o profissional da Saúde, mais crítico ainda dentro de um centro cirúrgico onde o 
médico (tutor) não poderia lançar mão dos formatos tradicionais de interação com o modelo 3D. Nesse ponto, lançamos mão da Interação Natural como interface alternativa para manipulação dos modelos $3 \mathrm{D}$, dando origem a um novo módulo na ferramenta Arthron, desenvolvido para incorporar uma interface em IN baseada no Kinect.

A Fig.4 apresenta o modelo conceitual deste módulo através de um diagrama de casos de uso UML. As principais funcionalidades da interface em IN são exatamente as mesmas funcionalidades de controle dos objetos 3D, ou seja, Escolher, Rotacionar, Aumentar, Diminuir, Mudar e Parar a manipulação do objeto 3D.

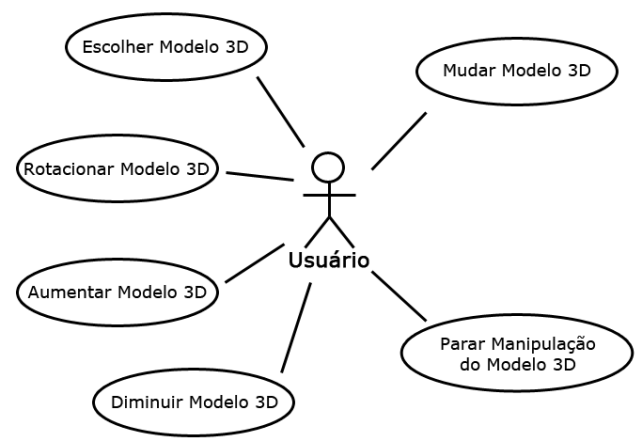

Figura 4. Diagramas de Casos de Uso UML para Módulo de IN da Arthron.

A Fig. 5 mostra a visão lógica da arquitetura de software desenvolvida onde destacamse os seguintes componentes: o Tracker, que reconhece os gestos e começa a rastrear a mão identificada e o Renderer, que recebe o rastreamento da mão e decide que manipulação exercer no objetos 3D.

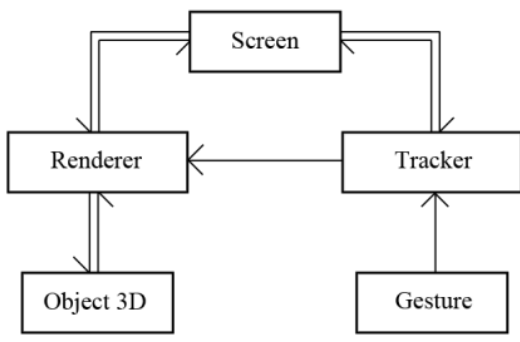

Figura 5. Visão Lógica da Arquitetura para Módulo de IN da Arthron.

O módulo de IN para Arthron foi desenvolvido usando o Swing de Java e o framework OpenNI (OpenNI Org, ___ ). O componente Tracker agrupa as classes HandTracker e HandTrackerHandler. HandTracker é a classe responsável pela utilização da biblioteca fornecida pelo framework OpenNI, onde é inicializado o Kinect e o rastreio das mãos e é feita a exibição do fluxo de imagem de profundidade. Para o início do rastreio é criado um evento que reconhece a ação de acenar que ativa o rastreio para a mão utilizada iniciando outros eventos, para criar, atualizar e destruir o rastreio. Esses últimos utilizam um objeto do tipo HandTrackerHandler para passar todas as atualizações relacionadas ao rastreio que são: criação de um rastreio, atualização do ponto onde se encontra a mão e que o rastreio foi perdido.

O componente Renderer corresponde a classe Renderer, que é uma implementação da interface HandTrackerHandler e tem uma instância passada como parâmetro na instanciação de um objeto do tipo HandTracker na classe Screen, fazendo assim que o 
objeto Renderer receba as atualizações relacionada aos rastreios. O objeto Renderer, ao receber a informação de um novo rastreio iniciado, salva o ponto inicial e caso não haja outro rastreio no momento define-o como a mão principal, se houver outra mão já sendo rastreada, é então definido como a mão secundária.

\section{Avaliação de Usabilidade}

Como observado anteriormente, a Telemedicina, e em especial a telementoria, permite a integração entre o paciente e a equipe que o acompanha, por meio de um ambiente virtual de troca de informações quando a distância separa os participantes. A mudança de paradigma da troca de informações através da interação física para a interação virtual implica na adaptação das pessoas envolvidas com o hardware e os sistemas de informação utilizados.

Pradhan (1994), citado por Castro et al. (2003), levanta questões básicas a respeito desses sistemas, como se é fácil de usar, que efeitos provocam nos cuidados ao paciente e qual a relação custo-benefício. Essas questões podem ser respondidas através da execução de uma etapa importante do processo de desenvolvimento de sistemas: a avaliação de usabilidade. Ela deve ocorrer paralelamente a esse processo para garantir e/ou verificar características como eficácia e segurança do sistema. No caso da telemedicina, pelo fato de representar uma nova modalidade para fornecer suporte às clínicas e pacientes, há o interesse em avaliar o impacto do sistema acerca do acesso, do custo e da qualidade do sistema (GRIGSBY et al., 2005).

A usabilidade de um sistema envolve fatores como facilidade de aprendizado, facilidade de uso, eficiência de uso e produtividade, satisfação do usuário, flexibilidade, utilidade e segurança no uso (Prantes e Barbosa, 2007). Uma deficiência em algum desses fatores pode se tornar uma barreira para o usuário e fazer com que ele se recuse a utilizar o sistema. A avaliação de um sistema pode determinar o seu grau de aplicabilidade e, além de detectar erros funcionais, obter o feedback do usuário final, visando reduzir as barreiras do uso do sistema.

Nesse sentido, foram realizadas três fases de teste para avaliação de usabilidade da interface em IN para manipulação de objetos 3D na Arthron. $O$ teste inicial da ferramenta de manipulação 3D serviu para acumular dados qualitativos. O perfil dos participantes foi definido de acordo com seu conhecimento das áreas de saúde e tecnologia, portando o público alvo dos testes foram estudantes de graduação dessas áreas. Quatro participantes foram usados para ajudar a testar o sistema, e todos eles foram filmados enquanto o faziam, além disso, há uma gravação de áudio juntamente com o questionário pós-teste.

O teste consistia no seguinte roteiro: escolha de modelo 3D, ativação de rastreamento de ambas as mãos, rotação do modelo $3 \mathrm{D}$, zoom do modelo $3 \mathrm{D}$, pausa nas manipulações do modelo 3D (botão "Stop") e pular para a tela inicial (botão "New Model"). Seguindo as instruções, o usuário irá escolher uma das mãos para acenar para o Kinect, depois a outra mão deve acenar para a ferramenta de interação natural, obtendo assim o rastreio de ambas as mãos. Se assim for desejado é possível acenar com as duas mãos ao mesmo tempo, permitindo assim uma utilização mais eficiente da aplicação, mas tal opção não é contemplada nas instruções pois poderia confundir o usuário principiante. 
A primeira mão rastreada é responsável pela manipulação da opção escolhida pela segunda mão. Ou seja, se o usuário escolher com sua segunda mão o botão "Zoom" o que definirá o quanto o objeto vai se aproximar ou se distanciar da tela serão os movimentos de aproximação ou distanciamento da primeira mão rastreada, em relação ao Kinect.

Seguindo o roteiro, o usuário deve mover o cursor designado a sua segunda mão rastreada para o botão "Rotation", e com a movimentação controlada pelo cursor designado a sua primeira mão rastreada, o usuário poderá rotacionar o modelo $3 \mathrm{D}$ escolhido. Para a segunda movimentação descrita pelo roteiro, a segunda mão irá selecionar o botão "Zoom" e modificar a visualização do modelo 3D de acordo com a movimentação da sua primeira mão rastreada. A Seleção do botão "Pause" independe da movimentação da primeira mão rastreada, pois a função de tal botão é somente manter a posição exata do modelo no momento em que tal botão foi ativado. Ao escolher o botão "New Model" o usuário volta à tela inicial.

Após o roteiro explicado acima, os participantes responderam quatro perguntas no questionário pós-teste: "A aplicação foi intuitiva?", "Você achou a aplicação agradável?", "Caso posto em uma situação em que você precisasse usar uma aplicação semelhante a esta, você se vê usando a mesma?", "Se você pudesse melhorar qualquer aspecto da aplicação, qual seria?".

No geral, os usuários definiram a aplicação como útil quando perguntados sobre alguma situação em que eles usariam a mesma. Cada participante tinha um diferente nível de conhecimento da ferramenta Kinect, porém todos conseguiram completar o teste com sucesso. Durante a avaliação foi chamada a atenção dos desenvolvedores para o posicionamento dos três botões da tela. Cada um dos botões controla uma ferramenta de manipulação. O zoom, a rotação e o stop, que desativa a manipulação do objeto na tela. Nessa segunda versão, Fig. 6 - (b), pode-se notar que o painel dos botões manipuladores mudou-se para a parte de baixo da interface, para diminuir o desgaste do usuário que prefira ficar com a mão perto das opções de manipulação.

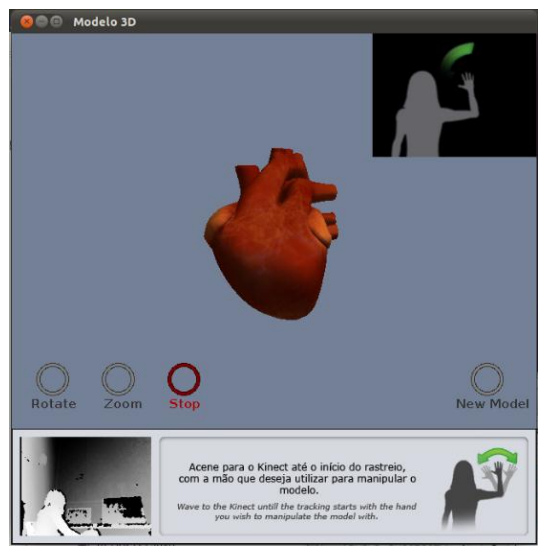

(a)

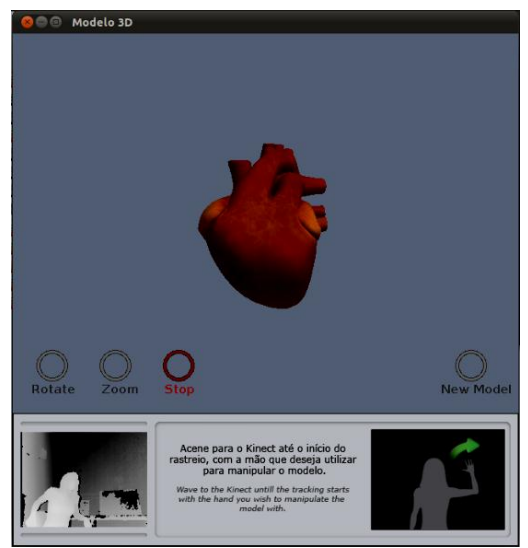

(b)

Figura 6. Versão da Interface de Usuário para utilização de IN na Arthron. Em (a) versão intermediária e em (b) versão atual da interface (pós avaliação). 


\section{Discussão dos Resultados}

Este trabalho apresenta resultados iniciais de uma experiência de desenvolvimento de uma interface que utiliza Interação Natural para a manipulação de objetos 3D em Telemedicina. Apesar de iniciais os resultados demonstram a aplicabilidade das técnicas de IN para telementoria em Telemedicina e, principalmente, os benefícios que elas podem trazer para área. Como principais contribuições do presente trabalho destacamos a facilidade do uso do Kinect para IN e os impactos da adoção dessa tecnologia para telementoria em Saúde.

O desenvolvimento para Interação Natural com o uso de Kinect é um recurso real e disponível que requer do desenvolvedor conhecimento das APIs disponíveis e livres e do hardware apropriado. Além disso, o âmbito lúdico da experiência cativa desenvolvedores e usuários no processo participativo de desenvolvimento. Nas pesquisas preliminares realizadas foi possível observar a grande vantagem no uso do Kinect, que permite o acesso à imagem de profundidade a preço acessível e tem disponível, via as bibliotecas existentes, tratamentos dessa imagem que utilizam pouco poder de processamento e fornecem informações que facilitam o desenvolvimento, como o rastreio de partes do corpo.

É possível concluir, então, que o advento e a popularização de interfaces mais sofisticadas que utilizam-se de Realidade Virtual e Interação Natural beneficiam áreas fins críticas como a Saúde. A telementoria em Saúde oferece várias vantagens aos tutores, médicos e aprendizes, no entanto, com o uso de facilidades de IN e RV é possível enriquecer ainda mais a experiência e praticamente trazer o aprendiz para dentro do centro cirúrgico. A experiência aqui apresentada ressalta a expansão virtual das salas de aprendizagem voltadas a Telemedicina e como a utilização de RV e IN pode tornar esses espaços mais colaborativos, interativos e imersivos. Por fim, salientamos que esta experiência abre novas perspectivas para o desenvolvimento e uso da Arthron, tais como: a expansão do uso de IN para outros controles da ferramenta (como chaveamento de fluxos) e a utilização de outras bibliotecas de objetos 3D.

\section{Agradecimentos}

Gostaríamos de agradecer a equipe do projeto GTAVCS e do Hospital Universitário Lauro Wanderley (UFPB) pela receptividade e colaboração para o desenvolvimento deste projeto.

\section{Referências}

Booch, G; Rumbaugh, J e Jacobson, I: UML, Guia do usuário: tradução; Fábio Freitas da Silva, Rio de Janeiro, Campus ,2000.

Carroll, John M. Human Computer Interaction (HCI). Denmark: The Interaction Design Foundation. . [Online] 2009. http://www.interactiondesign.org/encyclopedia/human_computer_interaction_hci.html.

Castro C. A. F. V., Gomes J. O., Vidal M. C. R. Questões relevantes para a ergonomia na usabilidade de sistemas de telemedicina. XXIII Encontro Nac. de Eng. de Produção - 2003.

Chapman, Glenn. 'Kinect' motion control for Xbox 360 makes magical debut. PHYS ORG. [Online] Junho 14, 2010. http://phys.org/news195709132.html. 
Grigsby J, Brega A. G., Devore P. A..The Evaluation of Telemedicine and Health Services Research. Telemed J E Health - 2005.

Kizony , Katz and Weiss. Adapting an immersive virtual reality system for rehabilitation. 2003, Vol. 14, pp. 261-268.

McCord, Charlotte. Gestures, Western Folklore. 1948. pp. 290-292. Vol. 7.

Miller, Joe. Wii speeds up the rehab process. USA Today. [Online] 2007. http://www.usatoday.com/tech/gaming/2007-07-24-wii-therapy_N.htm.

Nunes, F. L. S., Costa, R. M. E. M., Machado, L. S., Moraes, R. M.. Realidade Virtual para saúde no Brasil: conceitos, desafios e oportunidades. Revista Brasileira de Engenharia Biomédica Volume 27, Número 4, p. 243-258, 2011.

Oliveira,S. S.; Santos, B. A.; Vieira, E.; Passos, M. G.; Melo, E. A. G.; Motta, G. H. M. B.; Tavares, T. A.; Souza Filho, Guido Lemos de . Experiência de Uso da Arthron na Transmissão de Cirurgias em Tempo-Real para Telemedicina. In: XII Workshop de Informática Médica, 2012, Curitiba. Anais do XII WIM, 2012.

OpenNI Org. Introducing OpenNI. OpenNI Organization. [Online] http://www.openni.org/images/stories/pdf/OpenNI_UserGuide.pdf.

Sartori TCF, Fiorini VML. Programa de Orientação de Estudantes do Curso médico da FAMEMA: Resgatando, Reconstruindo e Buscando Bases para o Fortalecimento. Revista Brasileira de Educação Médica, no prelo, 2004.

Silva, J. C. F. ; Ferreira, A. ; Vieira, E. ; Passos, M. ; Melo, E. A. ; Tavares, T. A. ; Motta, G. H. M. B. ; Souza Filho, Guido Lemos de . A Multi-Stream Tool to Support Transmission in Surgery Applied to Telemedicine. In: International Workshop on Health and Social Care Information Systems and Technologies - HCIST2011, 2011, Algarve - Portugal. Proceedings of HCIST2011, 2011.

Strickland, Tremaine and Brigley. Team Uses Kinect to See Patient During Surgery. 2011.

Tanner, Lindsay. Doctors use Wii games for rehab therapy. USA Today. [Online] 2008. http://www.usatoday.com/tech/science/2008-02-08-wii-rehabilitation_N.htm.

Valli, Alessandro. Notes on Natural Interaction. 2007.

Vidal, Ângelo. Um Estudo Sobre a Utilização de Tecnologias de Interação Natural no Contexto de Fisioterapia. João Pessoa : s.n., 2011.

Yin, Ying and Davis, Randall. Toward Natural Interaction in the Real World: Real-time Yonk, Richard. The Age of the Interface. World Future Society. 2010. 